Voix et Images

voixetimages

\title{
Immensément poète : Anne Hébert
}

\section{André Brochu}

Volume 39, numéro 2 (116), hiver 2014

URI : https://id.erudit.org/iderudit/1025196ar

DOI : https://doi.org/10.7202/1025196ar

Aller au sommaire du numéro

Éditeur(s)

Université du Québec à Montréal

ISSN

0318-9201 (imprimé)

1705-933X (numérique)

Découvrir la revue

Citer ce compte rendu

Brochu, A. (2014). Compte rendu de [Immensément poète : Anne Hébert]. Voix et Images, 39(2), 153-158. https://doi.org/10.7202/1025196ar d'utilisation que vous pouvez consulter en ligne.

https://apropos.erudit.org/fr/usagers/politique-dutilisation/ 


\section{P O ÉS I E}

Immensément poète: Anne Hébert

$++$

\section{ANDRÉ BROCHU}

Université de Montréal

L'édition critique des Fuvres complètes d'Anne Hébert en cinq forts volumes, dans la prestigieuse (et hélas menacée) collection de la «Bibliothèque du Nouveau Monde», consacre l'intégralité de son premier tome à la poésie de la grande écrivaine ${ }^{1}$. On y trouve tous ses recueils publiés, depuis Les songes en équilibre (1942) jusqu'aux Poèmes pour la main gauche (1997), en plus d'une centaine de poèmes, soit parus en revue ou en d'autres imprimés mais non repris dans les recueils, soit inédits et appartenant à diverses périodes de sa vie. Ajoutons à cela une présentation critique élaborée du Dialogue sur la traduction à propos du "Tombeau des rois», qui contient la traduction anglaise par le poète Frank Scott, en trois versions, du plus célèbre poème d'Anne Hébert. Ce travail est le fruit d'un précieux échange épistolaire entre les deux écrivains.

Une édition critique ne mériterait pas son nom sans un appareil élaboré assurant l'établissement du texte, le commentaire historique, biographique, philologique et esthétique, et tout un ensemble de données concernant la réception du texte. C'est à cette tâche monumentale que s'emploie avec compétence Nathalie Watteyne, professeure de littérature à l'Université de Sherbrooke et responsable de l'ensemble du projet. En particulier, les rapprochements qu'elle effectue entre des textes de différentes époques, ou avec les sources d'inspiration d'Anne Hébert (Hector de SaintDenys Garneau et Charles Baudelaire en particulier), permettent une compréhension fine et plus globale de l'œuvre poétique. L'explication patiente de nombreux aspects relatifs au catholicisme, sans doute moins utile pour ceux qui ont mariné dans le Québec d'avant la Révolution tranquille, est au contraire indispensable pour les lecteurs des générations plus récentes, tant la religion et la référence biblique imprègnent l'inspiration de la poète malgré l'attitude critique qu'elle finit par adopter à l'égard de l'éducation reçue.

Certains lecteurs regretteront le format habituel des publications de la Bibliothèque du Nouveau Monde, qu'on pourrait qualifier de moyen. Ici, la dimension

1 Anne Hébert, Euvres complètes, t. 1 : Poésie, édition établie par Nathalie Watteyne, suivi de Dialogue sur la traduction à propos du "Tombeau des rois », édition établie par Patricia Godbout, Montréal, Presses de l'Université de Montréal, coll. «Bibliothèque du Nouveau Monde», 2013, 732 p. 
est nettement plus considérable. On imagine mal cependant faire tenir toute la matière en un plus petit ouvrage, à moins de le dédoubler. Soulignons que la taille des feuillets permet aisément l'inclusion des annotations critiques en bas de page plutôt qu'en fin de chapitre ou de section, inconvénient courant des éditions savantes.

Cela dit, certains problèmes demeurent, qui sont dus à d'autres aspects du protocole d'édition. Par exemple, on s'abstient de numéroter la première page d'un texte, ce qui est théoriquement défendable. Or, de très nombreux poèmes n'ont qu'une page, de sorte que leur succession entraîne l'absence de pagination sur de nombreux feuillets. C'est ainsi qu'on ne trouve aucune indication de page entre « Noël» (poème dont la deuxième et dernière page porte le chiffre 337) et «Que Dieu soit » (qui se termine à la p. 371)! Les numéros de pages apparaissant en haut, tantôt à gauche, tantôt à droite, il aurait été possible de disposer en bas au centre les numéros des textes d'une seule page, ou de trouver une autre solution. Tout, plutôt que le néant!

On constate ici les effets d'un dogmatisme qui est celui même de l'édition critique. Mais il y a plus grave, et cela concerne la succession même des recueils. Si, en principe, l'ordre chronologique doit être respecté, la règle s'applique aux œuvres pertinentes, c'est-à-dire celles qui justifient le projet d'une édition critique. Par conséquent, avoir installé en toute première place le long et problématique recueil intitulé Les songes en équilibre me semble une erreur. On sait que l'auteure a refusé toute sa vie de le laisser rééditer, pour des raisons qu'une lecture permet vite de comprendre. La poète débutante est encore loin d'y manifester la maîtrise de son inspiration, et fait montre plutôt d'une immaturité qui est aux antipodes de son art à venir. Certes, il fallait inclure ce recueil dans le livre, mais il aurait beaucoup mieux figuré en appendice, au titre de tentative méritoire mais incomplète. Certains éléments des Songes en équilibre annoncent sans doute les réussites futures, mais d'autres, d'une grande naïveté, frisent la caricature. Par exemple, ce début d'un «Poème pour papa »: "Ô mon père,/Ô mon ami,/Ô mon petit enfant!» (174) Ou encore, ces vers adressés à sa sœur Marie : «Dors, mon petit Pitou,/Mon beau trésor,/Dors, dors!» (177) La sensiblerie à tonalité familiale rejoint les effusions d'un grand nombre de poèmes religieux («Prière», «Communion», «Sainte Vierge Marie», «La Sainte-Vierge m’a parlé»...) qu'auraient pu signer les religieuses de cette époque très cléricale. Les songes en équilibre auraient pu accompagner en fin de recueil les Poèmes publiés non repris dans les recueils et les Poèmes inédits, eux aussi écartés par l'auteure. Ces derniers sont d'ailleurs, très souvent, d'une facture supérieure à celle des poèmes « de jeunesse».

Le passage des Songes en équilibre au Tombeau des rois, publié onze ans plus tard (1953), correspond à une véritable révolution de la manière hébertienne. À la maladresse des débuts, à la mièvrerie catholique succède une exploration des profondeurs intimes dont notre littérature ne présentait jusque-là aucun équivalent. Et cette transformation remarquable de la thématique et de la forme - on passe d'une poésie bavarde, voire facile et répétitive, à un discours d'une rigueur et d'une nouveauté extrêmes - constitue l'acte fondateur d'une œuvre qui, miraculeusement, comportera tout de suite une autre surprise. En effet, la distance est aussi grande entre les deux premiers recueils qu'entre Le tombeau des rois et Mystère de la parole, publié sept ans plus tard (1960). Certes, il ne s'agit plus de différence de qualité, celle-ci étant aussi élevée dans les deux cas, mais de métamorphose du contenu poétique: passage du 
vers bref au vers ample, de la quête du moi à la célébration du monde, de l'affrontement de la nuit intérieure à l'exploration de l'être. On peut ajouter à cela une rhétorique souvent plus hermétique dans Mystère de la parole, malgré l'optimisme affiché et la métrique déliée (vers long). Certaines apparences peuvent laisser croire à une atténuation du motif du désespoir, si présent dans Le tombeau des rois, mais il ne faut pas, malgré la métamorphose du discours poétique, surestimer l'importance des contrastes. Le lyrisme, en effet, gagne en complexité; et la rhétorique, en violence. Voici, par exemple, une évocation du destin d'Ève, mère du genre humain, qui se confond ici avec la mère du Christ: «Vois tes fils et tes époux pourrissent pêle-mêle entre tes cuisses, sous une seule malédiction/Mère du Christ souviens-toi des filles dernières-nées, de celles qui sont sans nom ni histoire, tout de suite fracassées entre deux très grandes pierres » (313). Le carnage devient la représentation par excellence de la vie, au plus près de ses sources divines.

On peut noter en passant que les figures bibliques, d'une orthodoxie irréprochable dans Les songes en équilibre, deviennent l'objet d'inventions étonnantes, et parfois même scabreuses, dans les grands recueils.

En somme, peu d'écrivains auront su, dans leurs premières œuvres, parcourir de telles distances signifiantes et renouveler si profondément leur problématique, tant formelle que thématique.

Sans doute le temps est-il le garant de ces progrès. Quand elle publie son premier recueil, Anne Hébert a vingt-six ans et n'est donc plus (malgré la complaisance enfantine de nombreuses pièces) une jeune fille. Onze ans puis sept ans serviront de tremplin aux magnifiques accomplissements futurs. Les poèmes ultérieurs (Le jour n'a d'égal que la nuit, Poèmes pour la main gauche) feront appel aux styles ou aux façons déjà établis: plus de révolution, mais l'exploitation de modes d'écriture qui ont fait leurs preuves. On peut certes sentir quelque affaiblissement de la ferveur poétique, au profit d'une œuvre narrative en plein essor, et comprendre par là les trente-deux ans qui séparent la publication de Poèmes (qui réunit Le tombeau des rois et Mystère de la parole) de Le jour n'a d'égal que la nuit. Paru en 1992, cet ouvrage a davantage les allures d'une collection de poèmes inédits - poèmes réussis, bien entendu, mais conçus séparément - que d'un recueil à proprement parler. Sa composition, qui aligne des "poèmes anciens (1961-1980)» (pourtant postérieurs à Mystère de la parole) et des "poèmes nouveaux (1987-1989)", a quelque chose d'étranger aux perspectives de la thématique d'ensemble, d'où l'impression qu'ils peuvent engendrer, de pièces rapportées, voire de collage de morceaux divers. Certains de ceux-ci sont relativement simples et accessibles, même s'ils ne versent pas dans la facilité des Songes en équilibre; d'autres retrouvent la logique énigmatique de Mystère de la parole.

Quant aux Poèmes pour la main gauche (1997), ils renouent davantage avec une poétique du recueil, mais sans renouveler en profondeur le discours du poème. On ne peut qu'en dire autant de la centaine de poèmes non repris en recueil ou inédits.

Plusieurs des titres de poèmes d'Anne Hébert sont constitués de simples noms communs ou de brefs énoncés centrés sur un substantif, et cela, dès le premier recueil : «Jour de juin», «Les deux mains», «Soir», «Figure de proue», «Sous la pluie», «Musique», «Danse», «Instant», «Fontaine», «Le vent», etc. Ce sont là des aspects 
du monde - temps ou espace, choses de la nature, activités humaines, objets culturels - dont la signification est immédiatement perceptible, des sortes de lieux communs de l'expérience quotidienne. Beaucoup de ces réalités extérieures pourraient évoquer des sujets de composition scolaire: «Pluie», «Amour», «Noël», «Le piano», «La page blanche» (Le jour n'a d'égal que la nuit). On perçoit là un côté de l'inspiration de l'auteure: sa simplicité voulue, ou peut-être même spontanée. Spontanée, elle l'est certes dans Les songes en équilibre, où le poème s'emploie le plus souvent à développer une idée à grand renfort de répétitions de mots ou de structures syntaxiques, par exemple dans «La chambre d'enfant» où

\author{
Des voix vous accueillent, \\ Bonnes et familières. \\ L'une vous dit, rassurante: \\ - Je suis le chien \\ Qui garde la maison... - \\ Une autre reprend, lointaine: \\ - Je suis le chat \\ Qui garde le feu... \\ Et moi, le grillon \\ Qui ensorcelle la maison. (171)
}

L'univers du conte, familier à tous les enfants, sert ici de toile de fond.

D'une certaine façon, une même orientation va dicter le choix des sujets dans les grands recueils - et en ce sens, Les songes en équilibre font bel et bien partie de l'univers poétique d'Anne Hébert. Sauf que le sujet, très sobre au départ, par exemple «Neige» (Mystère de la parole), donne lieu à un développement d'une grande complexité, tant de l'idée que de l'image:

\footnotetext{
La neige nous met en rêve sur de vastes plaines, sans traces ni couleur Veille mon cœur, la neige nous met en selle sur des coursiers d'écume Sonne l'enfance couronnée, la neige nous sacre en haute mer, plein songe, toutes voiles dehors [...] (303)
}

«La neige nous met en rêve » : la formulation est inusitée, le rêve apparaît comme un prolongement de l'univers naturel, prolongement régi par lui qui se détaille en vastes plaines, et ces plaines sont données pour abstraites, «sans traces ni couleur». C'est une façon d'indiquer que la neige, malgré sa blancheur, rejoint l'absence de couleur qui est celle du noir, les contraires s'épousent sourdement. S'épousent aussi le décor naturel et la conscience affective («mon cœur»), le moi aussitôt se déploie en un «nous» lancé dans une cavalcade sur les coursiers d'écume, neige et mer se confondent, terre et mer aussi... et l'on pourrait s'employer longtemps à démêler les connotations qui se présentent à travers les fastes du langage.

Voilà qui caractérise le discours de la poète à compter du Tombeau des rois. Elle part du plus simple pour s'élever aussitôt vers d'inimaginables régions signifiantes. 
L'invention succède à l'invention, tout se mêle sans pourtant sombrer dans la confusion. Le poème par excellence d'Anne Hébert, "Le tombeau des rois», raconte une descente au cœur de la nuit qui évoque simultanément, de façon très condensée, les mythologies grecque (Ariane, le dédale), égyptienne (les pharaons d'ébène) et chrétienne (la visite aux enfers se terminant par un retour au jour qui rappelle la résurrection du Christ après son séjour aux enfers). L'expérience du moi confronté à ses plus intimes profondeurs rejoint ainsi l'expérience cruciale de l'humanité vécue à travers ses représentants les plus illustres. Et qui plus est, c'est une femme, alter ego de l'auteure munie des seuls pouvoirs de l'écriture, qui reprend et mène à son terme l'aventure.

Entre le langage univoque des Songes en équilibre et celui des grands recueils - il faut constater toutefois que de nombreux passages du premier manifestent déjà une tendance vers un discours plus créateur -, des poèmes inédits ou publiés en revue et non repris dans les recueils, poèmes antérieurs à 1950, permettent de comprendre la transformation qui s'effectue au moment de la rédaction du Tombeau des rois. Certes, si l'auteure les a écartés, de même que de nombreux textes ultérieurs, c'est que, malgré leur richesse au moins relative, ils n'arrivaient pas à cette perfection qu'elle recherchait dans la conception ou la formulation. Prenons l'exemple de cet inédit charmant écarté de Poèmes pour la main gauche:

\author{
DES OISEAUX RARES \\ Des oiseaux rares \\ Qu'on enferme pour la nuit \\ Rayonnent à petit feu \\ Sous la noire couverture \\ Leur chant qui ne dépasse pas le cœur obscur \\ Éclaire le chemin tout alentour \\ Sourdement \\ Comme braise dans les ténèbres (568)
}

Une tension est créée entre un mouvement vers le dedans (enfermement, sous la couverture, cœur obscur) et un mouvement vers le dehors (rayonnement, chant, clarté, braise). Et le rayonnement se fait malgré l'enfouissement «sous la noire couverture», et le chant ne dépasse pas le cœur obscur mais éclaire pourtant le chemin, et l'éclaire « sourdement»... Ici comme en tant d'autres poèmes qui obéissent à la précise logique hébertienne, les contraires se touchent et posent ensemble la question de l'intime et de ses rapports avec l'extérieur, de la nature et de ses rapports avec l'humain. Mais la confrontation des contraires est toujours périlleuse, et l'auteure a pu juger qu'elle manquait ici de consistance, ou frôlait l'arbitraire.

En complément aux poèmes d'Anne Hébert, on peut lire la traduction que Frank Scott, universitaire et poète canadien-anglais, a faite du «Tombeau des rois ». Elle s'accompagne des réflexions d'Anne Hébert et de Frank Scott sur la traduction, notamment celle de la poésie, qui soulève des complications difficiles à résoudre. On trouve aussi une préface du grand critique Northrop Frye, traduite par Jean Simard, et une «Note explicative» de Jeanne Lapointe, professeure à l'Université Laval. Anne 
Hébert, qui fait état d'une connaissance limitée de la langue anglaise, n'en commente pas moins avec beaucoup de précision chacun des vers traduits par Frank Scott, ce qui amène ce dernier à remanier profondément son texte. C'est ainsi que «The Tomb of the Kings » connaîtra plusieurs versions successives.

En français, bien entendu, il n'y en a qu'une, mais chaque lecteur peut et doit aménager les significations au meilleur de son entendement. 